\title{
Factors Associated with High Weight Gain and Obesity Duration: The Norwegian Women and Cancer (NOWAC) Study
}

\author{
Marisa da Silva ${ }^{a} \quad$ Elisabete Weiderpass ${ }^{a}$ b, c, d Idlir Licaja, e \\ Charlotta Rylander ${ }^{a}$ \\ a Department of Community Medicine, Faculty of Health Sciences, UiT The Arctic University \\ of Norway, Tromsø, Norway; ${ }^{b}$ Department of Research, Cancer Registry of Norway, Institute \\ of Population-Based Cancer Research, Oslo, Norway; ' ${ }^{C}$ Department of Medical Epidemiology \\ and Biostatistics, Karolinska Institutet, Stockholm, Sweden; ${ }^{\mathrm{d}}$ Genetic Epidemiology Group, \\ Folkhälsan Research Center, Helsinki, Finland; ${ }^{~}$ Clinical Research Department, Centre \\ François Baclesse, Caen, France
}

\section{Keywords}

Longitudinal studies $\cdot$ Obesity $\cdot$ Body weight changes $\cdot$ Risk factors

\begin{abstract}
Aim: To identify factors associated with high weight gain and obesity duration in a representative sample of Norwegian women. Methods: 66,618 Norwegian women aged 34-70 years at baseline were included in the analysis. Baseline and follow-up questionnaires completed in 1991-2011 provided information on height, weight as well as sociodemographic, lifestyle and reproductive factors. We assessed the association with multivariable logistic regression. $\boldsymbol{R e}-$ sults: Women gained on average $0.5 \mathrm{~kg} /$ year $(95 \% \mathrm{Cl} 0.5-0.5 \mathrm{~kg} /$ year) during 6 years of follow-up, and 3.5\% maintained in obesity during 13 years of follow-up. The factors with strongest association with high weight gain ( $\geq 10 \mathrm{~kg}$ ) were smoking cessation (cessation vs. no change, $\mathrm{OR}=4.39,95 \% \mathrm{Cl} 3.91-4.94$ ) and decreased physical activity level (decrease vs. no change, $\mathrm{OR}=2.40,95 \% \mathrm{Cl} 2.21-2.61$ ). Low physical activity level (high vs. low, $\mathrm{OR}=0.17,95 \%$ $\mathrm{Cl}$ 0.14-0.20), higher than median age at menarche (over median vs. median or under median, $\mathrm{OR}=0.36,95 \% \mathrm{Cl} 0.31-0.41$ ), and less than 10 years of education ( $>12$ years vs. $<10$ years, OR $=0.44,95 \% \mathrm{Cl} 0.37-0.51$ ) were strongly associated with obesity duration. Conclusion: The modifiable factor with the strongest association with adverse weight development and potential for prevention was low or decreased physical activity level.
\end{abstract}

(C) 2018 The Author(s)

Published by S. Karger $\mathrm{GmbH}$, Freiburg

Marisa da Silva

Department of Community Medicine, Faculty of Health Sciences

UiT The Arctic University of Norway

P.O. Box 6050 Langnes, 9037 Tromsø, Norway

marisa.e.silva @ uit.no 
da Silva et al:: Factors Associated with High Weight Gain and Obesity Duration: The Norwegian Women and Cancer (NOWAC) Study

\section{Introduction}

During the past 4 decades, the prevalence of obesity worldwide has surpassed the prevalence of underweight [1]. The increase in obesity prevalence is of great public health concern, as obesity and weight gain are independently associated with several negative health outcomes such as higher all-cause and cause-specific mortality, increased risk of cardiovascular disease, diabetes, and several types of cancer [2-8]. Long-term obesity (referred to as obesity duration) has also been associated with an increased risk of diabetes and obesity-related cancers $[9,10]$. However, factors associated with weight gain and obesity duration, and the interplay between these, are less clear. To date, most studies of factors associated with body weight have been cross-sectional; however, to understand the increasing trends of obesity we also need to study factors associated with body weight development, and then a longitudinal study design with individual-level data is preferred. The latest regional health examination in Norway reported a prevalence of obesity of $23.1 \%$ in women in 2006-2008, which was a $10 \%$ point increase when compared with data from 1984-1986 [11]. In addition, Statistics Norway conducts a survey on living conditions every 3 years in a representative sample of inhabitants in Norway aged 16 years or older. Since 1998 , the self-reported prevalence of obesity has increased in both women and men in Norway and reached $11 \%$ in women in 2015 . Although there are differences in obesity prevalence according to age groups, region, rural/urban settlements, and reporting method (selfreport or examination), the prevalence and increasing trends in obesity demonstrate that weight gain and obesity are major public health problems also in Norway. A deeper understanding of weight development and its underlying factors in various populations is needed to implement effective public health actions that could help control the obesity epidemic. In the present study, we assessed anthropometrics as well as sociodemographic, reproductive and lifestyle factors and the association with high weight gain and obesity duration. Additionally, we described short-term weight change and long-term BMI status among Norwegian women.

\section{Material and Methods}

Study Design, Participants, and Subcohorts

The Norwegian Women and Cancer (NOWAC) study is a nationally representative, population-based cohort study initiated in 1991. Women in NOWAC were randomly sampled from the Norwegian Central Population Register, which includes all Norwegian inhabitants. Details on the design, material, and procedures of the NOWAC study have been described elsewhere [12]. The NOWAC study was approved by The Regional Committee for Medical Research Ethics and The Norwegian Data Inspectorate, and all women provided written informed consent.

89,749 women, who returned a baseline questionnaire (Q1) and a follow-up questionnaire (Q2) 5-8 years later, were considered eligible for inclusion. Of these, 47,526 additionally returned a second follow-up questionnaire (Q3) 5-8 years after Q2. Women who returned Q2 were younger, weighed less, and were less likely to use hormone therapy (HT), compared to women who only returned Q1. Moreover, women who returned Q3 were also younger, weighed less, were less likely to use HT, and, further, had more years of education and were more likely to use oral contraceptives (OC), compared to women who only returned Q2.

We excluded 4 women with implausible values of weight $(<30$ or $>200 \mathrm{~kg})$ or height $(<100 \mathrm{or}>230 \mathrm{~cm})$ in any of the questionnaires and women who had missing values of weight or height at Q1 or Q2 $(n=3,429)$. Women with missing information on important covariates at baseline $(n=19,698)$ were also excluded. Thus, our final analytical sample consisted of 66,618 women aged 34-70 years at baseline. Analyses of i) weight change and high weight gain and ii) duration of BMI status and obesity duration were carried out in subcohorts of the final analytical sample. In analyses of weight change and high weight gain, we excluded an additional 5,707 women with missing follow-up information on physical activity. There were no women with missing follow-up information on smoking status and menopausal status, which together with physical 
da Silva et al.: Factors Associated with High Weight Gain and Obesity Duration: The Norwegian Women and Cancer (NOWAC) Study

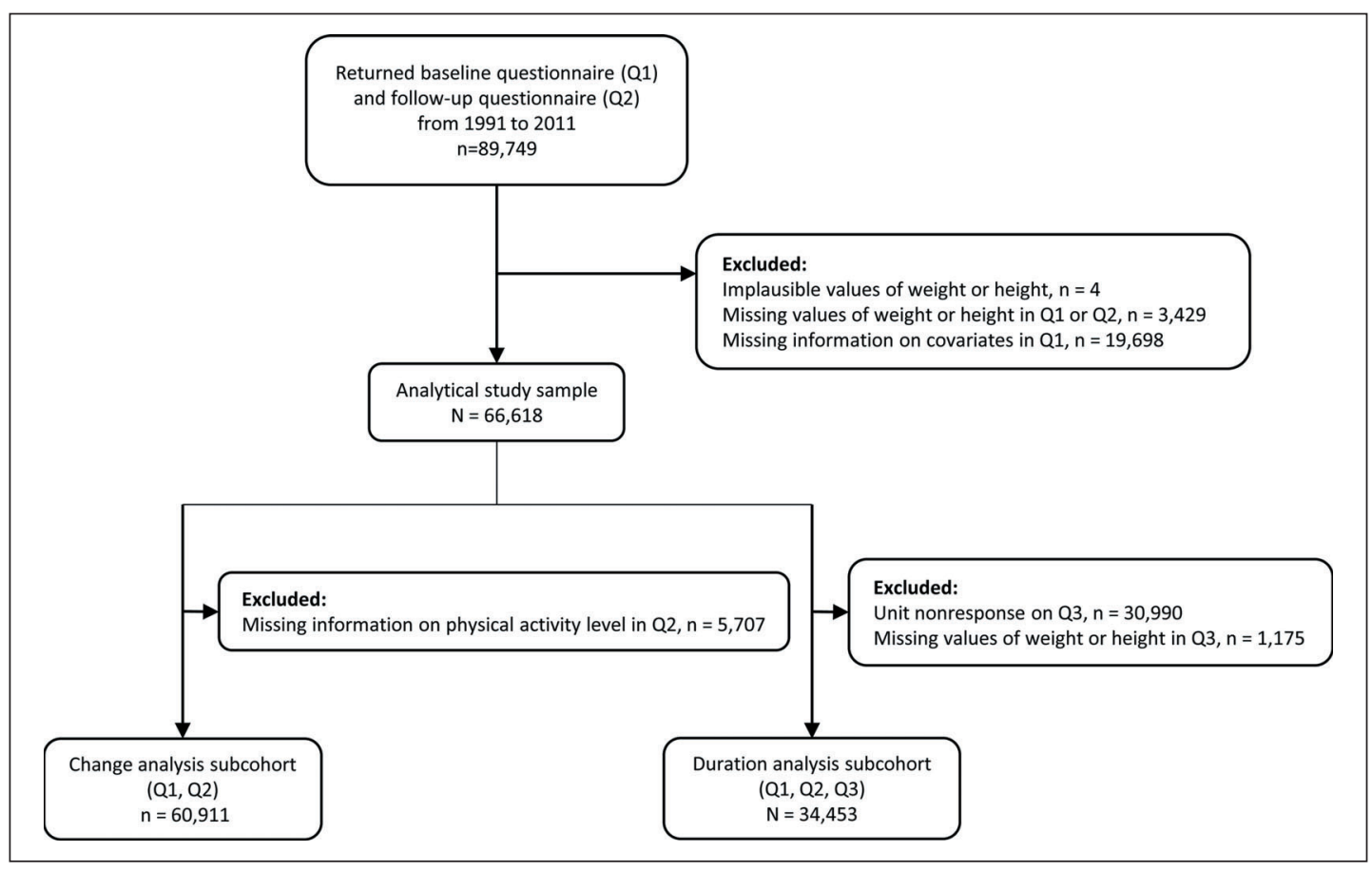

Fig. 1. Flowchart of study participants.

activity were the important transition factors. For analyses of duration of BMI status and obesity duration we excluded women who did not return Q3 $(n=30,990)$ or had missing information on weight or height in Q3 (n = 1,175) (fig. 1).

\section{Outcome Measures and Covariates}

Body weight change is commonly measured by either weight change in kilograms or BMI change, as BMI is a reasonably good measure of adiposity on a population level [13]. However, adults with stable height tend to follow upward weight trajectories, leading to increases in BMI until they reach the oldest age categories, when height decreases [14]. Weight change in kilograms tends to capture increases in fat mass more precisely than BMI change [13] and is also a more intuitive concept that can be communicated more effectively in public health recommendations. In order to capture short-term weight gain as long-term weight gain is more prone to weight cycling, we used self-reported weight from Q1 and Q2 to calculate weight change in kg. Weight change was categorized into five groups: weight loss $<-2 \mathrm{~kg})$, stable weight $(-2$ to $<2 \mathrm{~kg})$, low weight gain $(2$ to $<5 \mathrm{~kg}$ ), moderate weight gain $(5$ to $<10 \mathrm{~kg}$ ), or high weight gain ( $\geq 10 \mathrm{~kg})$. The average absolute weight change was calculated by subtracting the weight in Q1 from the weight in Q2, and the average annual weight change was calculated by dividing the absolute weight change by years of follow-up time between Q1 and Q2. BMI was calculated as self-reported weight in kilograms divided by the square of self-reported height in meters and categorized according to the World Health Organization definition [15]: underweight (BMI < $18.5 \mathrm{~kg} / \mathrm{m}^{2}$ ), normal weight (BMI 18.5 to $<25 \mathrm{~kg} / \mathrm{m}^{2}$ ), overweight (BMI 25 to $<30 \mathrm{~kg} / \mathrm{m}^{2}$ ), or obesity (BMI $\geq$ $30 \mathrm{~kg} / \mathrm{m}^{2}$ ). Duration of BMI status was defined as women who maintained in the same BMI category in Q1, $\mathrm{Q} 2$, and Q3. Thus, obesity duration is defined as women who maintained in the obesity category in all three questionnaires.

Potential factors associated with the outcomes were selected based on a priori knowledge and, unless stated, extracted from Q1. In the multivariable analyses we assessed sociodemographic factors such as age (5-year increments) and education ( $<10$ years / 10-12 years / >12 years). Lifestyle factors assessed were physical activity level based on an ordinal scale of 1-10 and collapsed into three categories: low $(\leq 4)$, moderate (5-6), or high ( $\geq 7)$, smoking status (never/former/current) and alcohol intake $(\leq \mathrm{median} /$ $>$ median g/day). Further, we assessed reproductive factors such as menopausal status (pre-/peri-/post- 
menopausal/unknown) as per definition in the Million Women Study [16], age at menarche ( $\leq$ median / $>$ median), parity (nulliparous / 1-2 children / $\geq 3$ or more children), OC use (never/ever), and HT use (never/ former/current). In addition, for analyses of weight change we assessed BMI status at baseline (underweight / normal weight / overweight / obesity) and the transition factors from Q1 to Q2: i) physical activity level (no change / increase / decrease), ii) smoking status (no change / restart / cessation) with the small number of smoking initiators $(n=63)$ merged together with 'no change', and iii) menopausal status (no or unknown transition to menopause / transition to menopause).

\section{Statistical Analyses}

Characteristics of weight change and duration of BMI status were assessed using chi-square tests for categorical variables and one-way ANOVA or the Kruskal-Wallis test for continuous variables. We used multivariable logistic regressions to assess factor's association with high weight gain and obesity duration. The regression models were built according to the 'purposeful selection' approach [17]. Briefly, we performed univariable regressions for each covariate and included those significant at a $20 \%$ level in the multivariable model (the full model). Next, we excluded covariates that were no longer significant in the full model using Wald statistics. Log-likelihood tests were performed to compare goodness of fit between the reduced model and the full model. Finally, we tested for biological plausible interactions. The reduced final model is presented in tables 4 and 5, with the excluded covariates presented in the footnotes. All statistical analyses were performed using STATA version 14.0 (Stata Corp., College Station, TX, USA).

\section{Results}

\section{Weight Change and Long-Term BMI Status}

In total, 60,911 women were included in the weight change analyses: mean \pm standard deviation (SD) age, weight and BMI was $46.1 \pm 8.1$ years, $65.5 \pm 10.7 \mathrm{~kg}$ and $23.6 \pm 3.6 \mathrm{~kg} / \mathrm{m}^{2}$, respectively. The average follow-up time from Q1 to Q2 was $6.5 \pm 0.8$ years and did not differ substantially across weight change categories. Compared to other weight change categories, women who experienced high weight gain were younger, taller, more likely to be current smokers, premenopausal, nulliparous, ever users of OC and never users of HT (table 1). Moreover, between Q1 and Q2, they were more likely to stop smoking, decrease their physical activity level and transition to menopause. Women who lost weight were older, more likely to be overweight or have obesity, had lower education, lower physical activity level, lower alcohol intake, and were more likely to be former smokers, compared with the other weight change categories. Further, they were more likely to be postmenopausal, have three or more children, never use OC, use HT, increase their physical activity level, and restart smoking between Q1 and Q2. Overall, 28.4\% of women reported stable weight $(-2$ to $<2 \mathrm{~kg}$ ), while $62.3 \%$ reported weight gain ( $\geq 2 \mathrm{~kg}$ ), and $9.4 \%$ reported weight loss $(<-2 \mathrm{~kg})$. Women gained on average $3.1 \mathrm{~kg}$ between Q1 and Q2 (95 \%confidence interval (CI): 2.0-3.1), which equals an average of $0.5 \mathrm{~kg}$ per follow-up year (95\% CI 0.5-0.5 kg) (table 2). Young women (34-40 years) gained the most weight ( $0.6 \mathrm{~kg} /$ year, $95 \% \mathrm{CI} 0.6-0.6)$, while old women (61-70 years) gained the least weight $(0.1 \mathrm{~kg} /$ year, $95 \% \mathrm{CI} 0.1-0.1)$.

34,453 women were included in the analysis of BMI status duration from Q1, Q2, and Q3. The average follow-up time between Q1 and Q3 was $13.1 \pm 0.3$ years. Compared to the other BMI duration categories, women that maintained in obesity had lower education, lower physical activity level, lower alcohol intake, and were more likely to be postmenopausal, report lower age at menarche than median, and never use OC (table 3). Women who maintained in the normal-weight category were taller, had higher education, higher physical activity level, higher alcohol intake, and were more likely to be premenopausal and more often use OC than women in the other BMI duration categories. Over the 13-year study period (Q1-Q3), most women maintained in the normal-weight category $(46.4 \%)$, while $8.7 \%$ maintained in the overweight, $3.5 \%$ in the obesity, and $0.5 \%$ in the underweight category. Moreover, $40.9 \%$ 
da Silva et al.: Factors Associated with High Weight Gain and Obesity Duration: The Norwegian Women and Cancer (NOWAC) Study

Table 1. Population characteristics by weight change category between baseline questionnaire (Q1) and follow-up questionnaire (Q2). The Norwegian Women and Cancer study, 1991-2011 ( $\mathrm{n}=60,911)$

\begin{tabular}{|c|c|c|c|c|c|}
\hline & \multicolumn{5}{|c|}{ Weight change category, kg } \\
\hline & $\begin{array}{l}\text { Weight loss } \\
(<-2 \mathrm{~kg})\end{array}$ & $\begin{array}{l}\text { stable weight } \\
(-2 \text { to }<2 \mathrm{~kg})\end{array}$ & $\begin{array}{l}\text { low weight gain } \\
\text { ( } 2 \text { to }<5 \mathrm{~kg} \text { ) }\end{array}$ & $\begin{array}{l}\text { moderate } \\
\text { weight gain } \\
(5 \text { to }<10 \mathrm{~kg})\end{array}$ & $\begin{array}{l}\text { high weight } \\
\text { gain } \\
(\geq 10 \mathrm{~kg})\end{array}$ \\
\hline Number of women (\%) & $5,717(9.4)$ & $17,284(28.4)$ & $16,919(27.8)$ & $15,202(25.0)$ & $5,789(9.5)$ \\
\hline \multicolumn{6}{|l|}{ Baseline characteristics $^{a}$} \\
\hline Mean age, years (SD) & $49.0(8.6)$ & $47.8(8.6)$ & $45.8(7.8)$ & $44.4(7.1)$ & $43.4(6.7)$ \\
\hline Mean weight, kg (SD) & $72.3(13.5)$ & $64.7(10.2)$ & $63.3(9.3)$ & $65.3(9.9)$ & $68.3(11.7)$ \\
\hline Mean height, cm (SD) & $166.5(5.7)$ & $166.2(5.6)$ & $166.2(5.6)$ & $166.7(5.5)$ & $167.2(5.5)$ \\
\hline \multicolumn{6}{|l|}{ BMI, \% } \\
\hline Underweight & 1.0 & 2.7 & 3.2 & 2.2 & 2.4 \\
\hline Normal weight & 46.2 & 71.1 & 76.2 & 71.3 & 60.9 \\
\hline Overweight & 35.6 & 21.3 & 17.7 & 21.7 & 27.6 \\
\hline Obesity & 17.2 & 5.0 & 3.0 & 4.8 & 9.1 \\
\hline \multicolumn{6}{|l|}{ Years of education, $\%$} \\
\hline$<10$ & 26.9 & 22.4 & 20.3 & 20.5 & 23.2 \\
\hline $10-12$ & 24.5 & 23.1 & 23.2 & 25.2 & 25.6 \\
\hline$>12$ & 48.6 & 54.5 & 56.6 & 54.2 & 51.3 \\
\hline \multicolumn{6}{|l|}{ Physical activity level, \% } \\
\hline Low & 32.3 & 23.8 & 23.0 & 26.9 & 32.1 \\
\hline Moderate & 39.7 & 42.9 & 43.2 & 42.8 & 38.5 \\
\hline High & 28.1 & 33.3 & 33.9 & 30.4 & 29.5 \\
\hline \multicolumn{6}{|l|}{ Smoking status, \% } \\
\hline Never smoker & 32.3 & 37.7 & 38.8 & 35.5 & 30.1 \\
\hline Former smoker & 34.1 & 33.2 & 32.9 & 31.9 & 29.6 \\
\hline Current smoker & 33.6 & 29.1 & 28.3 & 32.7 & 40.3 \\
\hline Median alcohol intake, g/day & 1.5 & 1.9 & 1.9 & 1.8 & 1.6 \\
\hline \multicolumn{6}{|l|}{ Menopausal status, $\%$} \\
\hline Premenopausal & 43.6 & 52.1 & 62.2 & 68.3 & 70.4 \\
\hline Perimenopausal & 5.3 & 4.8 & 4.3 & 4.2 & 3.8 \\
\hline Postmenopausal & 43.2 & 36.2 & 26.4 & 20.7 & 18.0 \\
\hline Unknown & 8.0 & 6.9 & 7.1 & 6.9 & 7.8 \\
\hline Mean age at menarche, years (SD) & $13.2(1.4)$ & $13.3(1.4)$ & $13.3(1.4)$ & $13.3(1.4)$ & $13.1(1.4)$ \\
\hline \multicolumn{6}{|l|}{ Parity, \% } \\
\hline Nulliparous & 9.2 & 8.8 & 8.6 & 8.4 & 9.7 \\
\hline $1-2$ children & 51.6 & 53.7 & 55.4 & 56.7 & 55.2 \\
\hline$\geq 3$ children & 39.2 & 37.5 & 36.1 & 34.9 & 35.1 \\
\hline \multicolumn{6}{|l|}{ Oral contraceptive use, $\%$} \\
\hline Never & 46.8 & 44.4 & 41.8 & 38.0 & 36.8 \\
\hline Ever & 53.2 & 55.6 & 58.3 & 62.0 & 63.2 \\
\hline \multicolumn{6}{|l|}{ Hormone therapy use, $\%$} \\
\hline Never & 77.1 & 80.0 & 84.2 & 86.9 & 87.7 \\
\hline Former & 9.2 & 6.5 & 5.0 & 4.4 & 4.3 \\
\hline Current & 13.7 & 13.5 & 10.8 & 8.7 & 8.1 \\
\hline
\end{tabular}

Table 1 continued on next page

changed their BMI status between questionnaires. Of these women, 79.8\% changed to a higher BMI category, $6.2 \%$ changed to a lower BMI category, and $14 \%$ cycled (data not shown).

\section{Factors Associated with High Weight Gain and Obesity Duration}

Smoking cessation displayed the strongestassociation with high weightgain, when compared to women in stable weight. Women who stopped smoking between Q1 and Q2 had more than four-fold higher odds of high weight gain (cessation vs. no change, $\mathrm{OR}=4.39,95 \% \mathrm{CI}: 3.91-4.94$ ). 
Table 1. Continued

Weight change category, kg

Weight loss stable weight low weight gain moderate high weight

$(<-2 \mathrm{~kg}) \quad(-2$ to $<2 \mathrm{~kg}) \quad(2$ to $<5 \mathrm{~kg}) \quad$ weight gain $\quad$ gain

Characteristics transition $Q 1 \rightarrow Q 2$

Physical activity level

\begin{tabular}{|c|c|c|c|c|c|}
\hline No change & 50.4 & 52.7 & 52.3 & 50.7 & 47.9 \\
\hline Increase & 28.8 & 23.9 & 21.6 & 20.7 & 16.7 \\
\hline Decrease & 20.9 & 23.3 & 26.1 & 28.7 & 35.3 \\
\hline \multicolumn{6}{|l|}{ loking status } \\
\hline No change & 87.7 & 90.1 & 88.5 & 84.6 & 77.1 \\
\hline Restart & 6.5 & 4.4 & 4.0 & 4.0 & 3.9 \\
\hline Cessation & 5.8 & 5.4 & 7.5 & 11.5 & 19.0 \\
\hline \multicolumn{6}{|l|}{ enopausal status } \\
\hline No or unknown transition to menopause & 52.1 & 43.8 & 34.2 & 28.3 & 26.7 \\
\hline Transition to menopause & 47.9 & 56.2 & 65.8 & 71.7 & 73.3 \\
\hline
\end{tabular}

$\mathrm{SD}=$ Standard deviation

${ }^{\mathrm{a}}$ Overall differences between weight change categories were significant for all variables $(\mathrm{p}<0.001)$.

Table 2. Average absolute and annual weight change in $\mathrm{kg}$ with $95 \%$ confidence interval (CI) by age group. The Norwegian Women and Cancer Study, 1991-2011

\begin{tabular}{|c|c|c|c|c|}
\hline & \multirow[t]{2}{*}{$\mathrm{n}$} & \multicolumn{3}{|c|}{ Follow-up time $\mathrm{Q} 1 \mathrm{Q} 1$ to $\mathrm{Q} 2, \mathrm{~kg}$, mean $(95 \% \mathrm{CI})^{\mathrm{a}}$} \\
\hline & & $\begin{array}{l}\text { to } Q 2 \text {, years }(95 \% \\
\text { CI) }\end{array}$ & $\begin{array}{l}\text { absolute weight } \\
\text { change }\end{array}$ & $\begin{array}{l}\text { annual weight } \\
\text { change }\end{array}$ \\
\hline \multicolumn{5}{|c|}{ Age group at baseline, years ${ }^{b}$} \\
\hline $34-40$ & 17,365 & $6.7(6.7-6.7)$ & $4.0(4.0-4.1)$ & $0.6(0.6-0.6)$ \\
\hline $41-50$ & 27,958 & $6.5(6.5-6.5)$ & $3.4(3.3-3.4)$ & $0.5(0.5-0.5)$ \\
\hline $51-60$ & 12,187 & $6.4(6.3-6.4)$ & $1.6(1.5-1.7)$ & $0.3(0.3-0.3)$ \\
\hline $61-70$ & 3,401 & $5.6(5.6-5.6)$ & $0.7(0.5-0.8)$ & $0.1(0.1-0.1)$ \\
\hline Total & 60,911 & $6.5(6.5-6.5)$ & $3.1(2.0-3.1)$ & $0.5(0.5-0.5)$ \\
\hline
\end{tabular}

Q1 = Baseline questionnaire; Q2 = follow-up questionnaire.

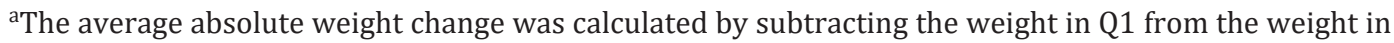
Q2, and the average annual weight change was calculated by dividing the absolute weight change by years of follow-up time between Q1 and Q2.

bverall differences between age groups were significant for absolute and annual weight change ( $<<$ $0.001)$.

Physical activity was also strongly associated with high weight gain, both the physical activity level in Q1 (high vs. low, OR $=0.30,95 \% \mathrm{CI} 0.27-0.33$ ) and a decrease in physical activity level between Q1 and Q2 (decrease vs. no change, OR = 2.40, 95\% CI 2.21-2.61). Further, already having obesity in Q1 was associated with a two-fold increase in odds of high weight gain (obesity vs. normal weight, $\mathrm{OR}=2.06,95 \% \mathrm{CI} 1.80-2.35$ ). Other factors significantly associated with high weight gain were being premenopausal which increased the odds of high weight gain, while young age, high education, being a current smoker, higher than median alcohol intake, and higher than median age at menarche decreased the odds of high weight gain (table 4). 
da Silva et al.: Factors Associated with High Weight Gain and Obesity Duration: The Norwegian Women and Cancer (NOWAC) Study

Table 3. Population characteristics by BMI duration category in baseline questionnaire (Q1), follow-up questionnaire (Q2) and second follow-up questionnaire (Q3). The Norwegian Women and Cancer Study, 1991-2010 ( $=34,453)$

\begin{tabular}{|c|c|c|c|c|c|}
\hline & \multicolumn{5}{|c|}{ BMI duration category, $\mathrm{kg} / \mathrm{m}^{2}$} \\
\hline & $\begin{array}{l}\text { stable } \\
\text { underweight }\end{array}$ & $\begin{array}{l}\text { stable normal } \\
\text { weight }\end{array}$ & $\begin{array}{l}\text { stable } \\
\text { overweight }\end{array}$ & stable obesity & not stable \\
\hline Number of women (\%) & $187(0.5)$ & $15,971(46.4)$ & $2,981(8.7)$ & $1,219(3.5)$ & $14,095(40.9)$ \\
\hline \multicolumn{6}{|l|}{ Baseline characteristics $^{a}$} \\
\hline Mean age, years (SD) & $41.7(4.6)$ & $42.1(5.1)$ & $44.5(5.1)$ & $44.4(5.3)$ & $42.5(5.1)$ \\
\hline Mean weight, kg (SD) & $48.3(4.0)$ & $59.1(5.4)$ & $73.3(5.8)$ & $91.3(10.5)$ & $66.2(9.0)$ \\
\hline Mean height, cm (SD) & $166.2(5.9)$ & $166.9(5.5)$ & $166.5(5.6)$ & $165.7(5.8)$ & $166.5(5.6)$ \\
\hline \multicolumn{6}{|l|}{ Years of education, $\%$} \\
\hline$<10$ & 15.0 & 14.7 & 23.2 & 27.2 & 21.3 \\
\hline $10-12$ & 23.0 & 22.4 & 25.3 & 27.2 & 26.0 \\
\hline$>12$ & 62.0 & 63.0 & 51.5 & 45.6 & 52.7 \\
\hline \multicolumn{6}{|l|}{ Physical activity level, \% } \\
\hline Low & 24.6 & 19.2 & 31.3 & 48.2 & 28.5 \\
\hline Moderate & 38.0 & 43.4 & 43.6 & 35.6 & 42.4 \\
\hline High & 37.4 & 37.5 & 25.1 & 16.2 & 29.2 \\
\hline \multicolumn{6}{|l|}{ Smoking status, \% } \\
\hline Never smoker & 40.6 & 37.1 & 39.3 & 40.1 & 34.3 \\
\hline Former smoker & 26.7 & 32.0 & 35.5 & 33.3 & 31.4 \\
\hline Current smoker & 32.6 & 30.9 & 25.2 & 26.6 & 34.4 \\
\hline Median alcohol intake, g/day & 1.6 & 1.9 & 1.6 & 0.9 & 1.7 \\
\hline \multicolumn{6}{|l|}{ Menopausal status, $\%$} \\
\hline Premenopausal & 77.5 & 79.6 & 68.2 & 67.6 & 77.0 \\
\hline Perimenopausal & 4.8 & 3.6 & 5.9 & 5.8 & 4.2 \\
\hline Postmenopausal & 13.4 & 9.8 & 16.2 & 17.9 & 10.8 \\
\hline Unknown & 4.3 & 7.0 & 9.8 & 8.7 & 8.0 \\
\hline Mean age at menarche, years (SD) & $13.9(1.4)$ & $13.4(1.4)$ & $13.0(1.3)$ & $12.7(1.4)$ & $13.2(1.4)$ \\
\hline \multicolumn{6}{|l|}{ Parity, \% } \\
\hline Nulliparous & 14.4 & 8.9 & 7.5 & 10.3 & 8.7 \\
\hline $1-2$ children & 61.0 & 59.2 & 54.4 & 51.9 & 56.4 \\
\hline$\geq 3$ children & 24.6 & 31.9 & 38.1 & 37.8 & 34.9 \\
\hline \multicolumn{6}{|l|}{ Oral contraceptive use, $\%$} \\
\hline Never & 36.9 & 33.9 & 41.3 & 46.2 & 35.8 \\
\hline Ever & 63.1 & 66.1 & 58.7 & 53.8 & 64.2 \\
\hline \multicolumn{6}{|l|}{ Hormone therapy use, \% } \\
\hline Never & 93.6 & 91.9 & 86.6 & 87.5 & 91.0 \\
\hline Former & 1.6 & 2.2 & 3.9 & 3.7 & 2.6 \\
\hline Current & 4.8 & 5.8 & 9.6 & 8.8 & 6.4 \\
\hline
\end{tabular}

$\mathrm{SD}=$ Standard deviation

${ }^{\mathrm{a}}$ Overall differences between BMI duration categories were significant for all variables $(\mathrm{p}<0.001)$.

Low physical activity level displayed the strongest association with maintaining in obesity compared to maintaining normal weight from Q1 to Q3 (high vs. low, OR $=0.17,95 \%$ CI 0.14-0.20). Higher than median age at menarche also decreased the odds of obesity duration (over median vs. median or under median, $\mathrm{OR}=0.36,95 \% \mathrm{CI} 0.31-0.41$ ) as well as high education ( $>12$ years vs. $<10$ years, $\mathrm{OR}=0.44,95 \% \mathrm{CI} 0.37-0.51$ ). In addition, being a current smoker, higher than median alcohol intake, ever use of OC significantly decreased the odds of obesity duration, while older age and being nulliparous significantly increased the odds of obesity duration (table 5). We found no evidence of biological plausible interactions for any of the models. 
da Silva et al.: Factors Associated with High Weight Gain and Obesity Duration: The Norwegian Women and Cancer (NOWAC) Study

Table 4. Factors associated with high weight gain $(n=5,789)$ compared to stable weight $(n=17,284)$, with univariable and multivariable odds ratios (OR) and 95\% confidence intervals (CI). The Norwegian Women and Cancer Study, 1991-2011

\begin{tabular}{|c|c|c|c|c|}
\hline & \multicolumn{4}{|c|}{ High weight gain $(\geq 10 \mathrm{~kg}$ ) } \\
\hline & \multicolumn{2}{|c|}{ univariable } & \multicolumn{2}{|c|}{ multivariable $^{\mathrm{a}}$} \\
\hline & OR & $95 \% \mathrm{CI}$ & OR & $95 \% \mathrm{CI}$ \\
\hline \multicolumn{5}{|l|}{ Factors } \\
\hline Age (5-year increments) & 0.70 & $0.69-0.72$ & 0.69 & $0.67-0.71$ \\
\hline \multicolumn{5}{|l|}{ BMI category $\left(\mathrm{kg} / \mathrm{m}^{2}\right)$} \\
\hline Underweight & 1.08 & $0.97-1.34$ & 0.87 & $0.71-1.08$ \\
\hline Normal weight & 1.00 & reference & 1.00 & reference \\
\hline Overweight & 1.55 & $1.44-1.66$ & 1.76 & $1.62-1.91$ \\
\hline Obesity & 2.14 & $1.90-2.40$ & 2.06 & $1.80-2.35$ \\
\hline \multicolumn{5}{|l|}{ Education } \\
\hline$<10$ years & 1.00 & reference & 1.00 & reference \\
\hline 10-12 years & 1.06 & $0.97-1.15$ & 0.92 & $0.84-1.02$ \\
\hline$>12$ years & 0.89 & $0.82-0.96$ & 0.78 & $0.71-0.85$ \\
\hline \multicolumn{5}{|l|}{ Physical activity level } \\
\hline Low & 1.00 & reference & 1.00 & reference \\
\hline Moderate & 0.66 & $0.62-0.71$ & 0.43 & $0.39-0.47$ \\
\hline High & 0.65 & $0.60-0.71$ & 0.30 & $0.27-0.33$ \\
\hline \multicolumn{5}{|l|}{ Smoking status } \\
\hline Never smoker & 1.00 & reference & 1.00 & reference \\
\hline Former smoker & 1.14 & $1.05-1.23$ & 1.13 & $1.03-1.23$ \\
\hline Current smoker & 1.77 & $1.64-1.90$ & 0.88 & $0.80-0.97$ \\
\hline \multicolumn{5}{|c|}{ Alcohol intake $($ median = $1.7 \mathrm{~g} /$ day $)$} \\
\hline$\leq 1.7 \mathrm{~g} /$ day & 1.00 & reference & 1.00 & reference \\
\hline$>1.7 \mathrm{~g} /$ day & 0.86 & $0.81-0.91$ & 0.85 & $0.79-0.90$ \\
\hline \multicolumn{5}{|l|}{ Menopausal status } \\
\hline Premenopausal & 2.65 & $2.45-2.86$ & 1.24 & $1.11-1.39$ \\
\hline Perimenopausal & 1.60 & $1.36-1.89$ & 1.09 & $0.91-1.31$ \\
\hline Postmenopausal & 1.00 & reference & 1.00 & reference \\
\hline Unknown & 2.25 & $1.98-2.56$ & 1.32 & $1.14-1.54$ \\
\hline \multicolumn{5}{|c|}{ Age at menarche (median $=13$ years) } \\
\hline$\leq 13$ years & 1.00 & reference & 1.00 & reference \\
\hline$>13$ years & 0.74 & $0.69-0.79$ & 0.88 & $0.82-0.94$ \\
\hline \multicolumn{5}{|l|}{ Transition factors, $Q 1 \rightarrow Q 2$} \\
\hline \multicolumn{5}{|l|}{ Physical activity level } \\
\hline No change & 1.00 & reference & 1.00 & reference \\
\hline Increase & 0.77 & $0.70-0.83$ & 0.47 & $0.43-0.52$ \\
\hline Decrease & 1.66 & $1.55-1.78$ & 2.40 & $2.21-2.61$ \\
\hline \multicolumn{5}{|l|}{ Smoking } \\
\hline No change & 1.00 & reference & 1.00 & reference \\
\hline Restart & 1.02 & $0.88-1.20$ & 0.69 & $0.58-0.82$ \\
\hline Cessation & 4.08 & $3.71-4.49$ & 4.39 & $3.91-4.94$ \\
\hline
\end{tabular}

Q1 = Baseline questionnaire; Q2 = follow-up questionnaire.

aEach variable was adjusted for all other variables shown in table. Potential covariates that did not reach statistical significance in the multivariable model were parity, oral contraceptive use, hormone therapy use and transition to menopause. 
da Silva et al.: Factors Associated with High Weight Gain and Obesity Duration: The Norwegian Women and Cancer (NOWAC) Study

Table 5. Factors associated with obesity duration $(n=1,219)$ compared to normal-weight duration $(n=$ 15,971), with univariable and multivariable odds ratios (OR) and 95\% confidence intervals (CI). The Norwegian Women and Cancer Study, 1991-2010

\begin{tabular}{|c|c|c|c|c|}
\hline & \multicolumn{4}{|c|}{ Obesity duration (Q1, Q2 and Q3) } \\
\hline & \multicolumn{2}{|c|}{ univariable } & \multicolumn{2}{|c|}{ multivariable $^{\mathrm{a}}$} \\
\hline & OR & $95 \% \mathrm{CI}$ & OR & $95 \% \mathrm{CI}$ \\
\hline \multicolumn{5}{|l|}{ Factors } \\
\hline Age (5-year increments) & 1.55 & $1.46-1.64$ & 1.49 & $1.39-1.59$ \\
\hline \multicolumn{5}{|l|}{ Education } \\
\hline$<10$ years & 1.00 & reference & 1.00 & reference \\
\hline 10-12 years & 0.66 & $0.56-0.77$ & 0.72 & $0.60-0.85$ \\
\hline$>12$ years & 0.39 & $0.34-0.45$ & 0.44 & $0.37-0.51$ \\
\hline \multicolumn{5}{|l|}{ Physical activity level } \\
\hline Low & 1.00 & reference & 1.00 & reference \\
\hline Moderate & 0.33 & $0.29-0.37$ & 0.33 & $0.29-0.38$ \\
\hline High & 0.16 & $0.14-0.19$ & 0.17 & $0.14-0.20$ \\
\hline \multicolumn{5}{|l|}{ Smoking status } \\
\hline Never smoker & 1.00 & reference & 1.00 & reference \\
\hline Former smoker & 0.96 & $0.84-1.10$ & 1.12 & $0.96-1.31$ \\
\hline Current smoker & 0.79 & $0.69-0.92$ & 0.83 & $0.70-0.97$ \\
\hline \multicolumn{5}{|c|}{ Alcohol intake $($ median $=1.9 \mathrm{~g} /$ day $)$} \\
\hline$\leq 1.9 \mathrm{~g} /$ day & 1.00 & reference & 1.00 & reference \\
\hline$>1.9$ g/day & 0.45 & $0.40-0.52$ & 0.48 & $0.42-0.56$ \\
\hline \multicolumn{5}{|c|}{ Age at menarche (median $=13$ years) } \\
\hline$\leq 13$ years & 1.00 & reference & 1.00 & reference \\
\hline$>13$ years & 0.39 & $0.34-0.44$ & 0.36 & $0.31-0.41$ \\
\hline \multicolumn{5}{|l|}{ Parity } \\
\hline Nulliparous & 1.33 & $1.09-1.62$ & 1.43 & $1.15-1.78$ \\
\hline $1-2$ children & 1.00 & reference & 1.00 & reference \\
\hline$\geq 3$ children & 1.36 & $1.20-1.54$ & 1.14 & $0.99-1.30$ \\
\hline \multicolumn{5}{|l|}{ Oral contraceptive use } \\
\hline Never & 1.00 & reference & 1.00 & reference \\
\hline Ever & 0.60 & $0.53-0.67$ & 0.79 & $0.70-0.91$ \\
\hline
\end{tabular}

Q1 = Baseline questionnaire; Q2 = follow-up questionnaire; Q3 = second follow-up questionnaire.

aEach variable was adjusted for all other variables shown in table. Potential covariates that did not reach statistical significance in the multivariable model were menopausal status and hormone therapy use.

\section{Discussion}

In the present study, we have described short-term weight change and long-term BMI status and identified factors associated with high weight gain and obesity duration in a representative, population-based Norwegian female cohort. Our results show that the mean adult body weight in Norwegian women increased during the study period and that younger women gained more weight than older, which is in accordance with other studies [18-21]. It is challenging to compare the mean annual weight change between studies, as population age distribution and follow-up time may differ considerably. A longer follow-up time and older population will result in a lower mean annual weight change, and vice versa. In accordance with our results, The Australian Longitudinal Study on Women's Health also reported an average annual weight gain of $0.5 \mathrm{~kg}$ with comparable follow-up time and mean population age [22]. 
da Silva et al.: Factors Associated with High Weight Gain and Obesity Duration: The Norwegian Women and Cancer (NOWAC) Study

Several lifestyle factors were significantly associated with high weight gain and obesity duration. Physical activity and smoking were the strongest lifestyle factors associated with high weight gain which is in agreement with findings from the Tromsø Study [23]. Low physical activity displayed the single strongest association with obesity duration, and a decrease in physical activity increased the odds of high weight gain more than two-fold. In the literature, the association between physical activity and prevention of weight gain has been inconsistent $[20,22,24,25]$, although there is strong agreement that physical activity can prevent obesity and lead to weight loss and other health benefits [26]. The relationship between smoking and the studied outcomes was complex, since it both increased and decreased the odds depending on smoking status or transition. Current smoking decreased the odds of obesity duration and high weight gain. However, smoking cessation between Q1 and Q2 displayed the strongest association with high weight gain, with four-fold increased odds, compared to women who did not change their smoking status. Smoking is associated with increased metabolic rate, decreased metabolic efficiency, and reduced appetite, but there are uncertainties regarding the effect of smoking on weight control [27]. On the other hand, cross-sectional studies have shown that heavy smoking is positively related to BMI [28, 29]. Smoking cessation is a well-established determinant of weight gain [27], and our result that smoking cessation was strongly correlated with weight gain is in accordance with those of other studies $[22,24]$. The possibility of gaining weight can hamper the motivation to quit smoking, which is worrying, since smoking increases the risk of cardiovascular disease and cancer [30]. However, immediate weight gain after smoking cessation tends to attenuate [24], and smoking cessation should always be recommended regardless of any possible short-term weight gain. The factor with the third strongest association with high weight gain was already having obesity. Among the other factors significantly associated with high weight gain, only alcohol intake was a modifiable factor. Other prospective studies have found similar results that alcohol intake was negatively associated with weight gain [20, 22, 23, 31]. However, alcohol intake is not an effective weight control measure and is associated with other negative health outcomes, such as increased risk of certain types of cancer [32]. To the best of our knowledge, there are no other studies on factors associated with obesity duration (here defined as long-term obesity) with detailed information on sociodemographic, reproductive, and lifestyle factors. Thus, it is difficult to compare our results on factors associated with obesity duration with others in the literature.

The main strength of our study is that it includes a large, representative, populationbased sample of Norwegian women. The comprehensive questionnaires enabled us to control for several covariates, and our longitudinal study design allowed us to use repeated measurements for outcome measures and transition covariates such as smoking, physical activity, and menopausal status. The importance of including transition variables in studies of weight change is exemplified in this study by smoking, as being a current smoker went from increasing to decreasing the odds of high weight gain after adjustment for smoking cessation. To the best of our knowledge, there has been no previous assessment of the average annual increase in body weight in a representative sample of women in middle adulthood in Norway. Nevertheless, this study has several limitations. Height and weight were self-reported, and there is a well-established tendency to underestimate height and weight, which increases with age and BMI [33]. A validation study of BMI in NOWAC was recently conducted and showed substantial agreement between self-reports and objective measurements values, although greater misclassification due to underreporting was observed in women with overweight and obesity [34]. Further, the physical activity scale in NOWAC was recently validated against a sensor that monitored heart rate and movement, showing a significant agreement but only moderate Spearman's rank correlation coefficients $(0.36-0.46, p<0.001)$ [35]. We had to omit total energy intake from the analyses as the food frequency questionnaire was not 
provided to all participants in this study, leading to a large amount of missing data, and because of known biases with respect to obesity [36]. However, the actual weight change could function as a proxy for positive energy imbalance in our weight change analyses.

In summary, over a period of 6 years of follow-up, women in middle adulthood gained on average $0.5 \mathrm{~kg}$ per year, and the largest increase in weight was among younger women. During 13 years of follow-up, 3.5\% of women maintained in the obesity category. Lifestyle factors such as smoking cessation, physical activity decrease, and already having overweight and obesity were strongly associated with high weight gain. While for obesity duration, low physical activity, higher than median age at menarche, and less than 10 years of education displayed the strongest associations. Accordingly, physical activity can contribute considerably to the prevention of adverse weight development among Norwegian women in middle adulthood.

\section{Acknowledgments}

The authors thank the staff and participants of the NOWAC study for their valuable contributions.

\section{Disclosure Statement}

The authors declare no competing interests.

\section{References}

$\checkmark 1$ NCD Risk Factor Collaboration: Trends in adult body-mass index in 200 countries from 1975 to 2014: a pooled analysis of 1698 population-based measurement studies with 19.2 million participants. Lancet 2016;387: 1377-1396.

-2 Lavie CJ, Milani RV, Ventura HO: Obesity and cardiovascular disease: risk factor, paradox, and impact of weight loss. J Am Coll Cardiol 2009;53:1925-1932.

-3 Abdullah A, Peeters A, de Courten M, Stoelwinder J: The magnitude of association between overweight and obesity and the risk of diabetes: a meta-analysis of prospective cohort studies. Diabetes Res Clin Pract 2010; 89:309-319.

4 Renehan AG, Tyson M, Egger M, Heller RF, Zwahlen M: Body-mass index and incidence of cancer: a systematic review and meta-analysis of prospective observational studies. Lancet 2008;371:569-578.

5 Whitlock G, Lewington S, Sherliker P, Clarke R, Emberson J, Halsey J, Qizilbash N, Collins R, Peto R: Body-mass index and cause-specific mortality in 900000 adults: collaborative analyses of 57 prospective studies. Lancet 2009;373:1083-1096.

6 Keum N, Greenwood DC, Lee DH, Kim R, Aune D, Ju W, Hu FB, Giovannucci EL: Adult weight gain and adiposityrelated cancers: a dose-response meta-analysis of prospective observational studies. J Natl Cancer Inst 2015; 107: doi: 10.1093/jnci/djv088.

7 Colditz GA, Willett WC, Rotnitzky A, Manson JE: Weight gain as a risk factor for clinical diabetes mellitus in women. Ann Intern Med 1995;122:481-486.

8 Kannel WB, D'Agostino RB, Cobb JL: Effect of weight on cardiovascular disease. Am J Clin Nutr 1996(3 suppl):419S-422S.

-9 Arnold M, Jiang L, Stefanick ML, Johnson KC, Lane DS, LeBlanc ES, Prentice R, Rohan TE, Snively BM, Vitolins M, Zaslavsky O, Soerjomataram I, Anton-Culver H: Duration of Adulthood Overweight, Obesity, and Cancer Riskin the Women's Health Initiative: a longitudinal study from the United States. PLoS Med 2016;13:e1002081.

10 Power C, Thomas C: Changes in BMI, duration of overweight and obesity, and glucose metabolism: 45 years of follow-up of a birth cohort. Diabetes Care 2011;34:1986-1991.

-11 Midthjell K, Lee CM, Langhammer A, Krokstad S, Holmen TL, Hveem K, Colagiuri S, Holmen J: Trends in overweight and obesity over 22 years in a large adult population: the HUNT Study, Norway. Clin Obes 2013;3: 12-20.

12 Lund E, Dumeaux V, Braaten T, Hjartaker A, Engeset D, Skeie G, Kumle M: Cohort profile: The Norwegian Women and Cancer Study - NOWAC-Kvinner og kreft. Int J Epidemiol 2008;37:36-41.

13 Hu F: Obesity Epidemiology. New York, Oxford University Press, 2008. 
da Silva et al.: Factors Associated with High Weight Gain and Obesity Duration: The Norwegian Women and Cancer (NOWAC) Study

14 Malhotra R, Ostbye T, Riley CM, Finkelstein EA: Young adult weight trajectories through midlife by body mass category. Obesity (Silver Spring) 2013;21:1923-1934.

15 WHO Expert Committee on Physical Status: Physical Status: the Use and Interpretation of Anthropometry. Geneva, World Health Organization, 1995. Report no.: 854.

16 Beral V: Breast cancer and hormone-replacement therapy in the Million Women Study. Lancet 2003;362: 419-427.

17 Veierød MB, Lydersen S, Laake P (eds): Medical Statistics in Clinical and Epidemiological Research. Oslo, Gyldendal akademisk, 2012.

18 Jacobsen BK, Aars NA: Changes in body mass index and the prevalence of obesity during 1994-2008: repeated cross-sectional surveys and longitudinal analyses. The Tromso Study. BMJ Open 2015;5:e007859.

19 Tanamas SK, Shaw JE, Backholer K, Magliano DJ, Peeters A: Twelve-year weight change, waist circumference change and incident obesity: the Australian diabetes, obesity and lifestyle study. Obesity (Silver Spring) 2014; 22:1538-1545.

20 El Reda D, Strom P, Sandin S, Oh JK, Adami HO, Lof M, Weiderpass E: Determinants of long-term weight change among middle-aged Swedish women. Obesity (Silver Spring) 2017;25:476-485.

21 Sheehan TJ, DuBrava S, DeChello LM, Fang Z: Rates of weight change for black and white Americans over a twenty year period. Int J Obes Relat Metab Disord 2003;27:498-504.

22 Brown WJ, Williams L, Ford JH, Ball K, Dobson AJ: Identifying the energy gap: magnitude and determinants of 5-year weight gain in midage women. Obes Res 2005;13:1431-1441.

23 Wilsgaard T, Jacobsen BK, Arnesen E: Determining lifestyle correlates of body mass index using multilevel analyses: the Tromso Study, 1979-2001. Am J Epidemiol 2005;162:1179-1188.

24 Mozaffarian D, Hao T, Rimm EB, Willett WC, Hu FB: Changes in diet and lifestyle and long-term weight gain in women and men. N Engl J Med 2011;364:2392-2404.

25 Lee IM, Djousse L, Sesso HD, Wang L, Buring JE: Physical activity and weight gain prevention. JAMA 2010;303: 1173-1179.

26 Bray GA, Fruhbeck G, Ryan DH, Wilding JP: Management of obesity. Lancet 2016;387:1947-1956.

27 Chiolero A, Faeh D, Paccaud F, Cornuz J: Consequences of smoking for body weight, body fat distribution, and insulin resistance. Am J Clin Nutr 2008;87:801-809.

28 Chiolero A, Jacot-Sadowski I, Faeh D, Paccaud F, Cornuz J: Association of cigarettes smoked daily with obesity in a general adult population. Obesity (Silver Spring) 2007;15:1311-1318.

29 John U, Hanke M, Rumpf HJ, Thyrian JR: Smoking status, cigarettes per day, and their relationship to overweight and obesity among former and current smokers in a national adult general population sample. Int J Obes (Lond) 2005;29:1289-1294.

30 Office on Smoking and Health National Center for Chronic Disease Prevention and Health Promotion: How Tobacco Smoke Causes Disease: The Biology and Behavioral Basis for Smoking-Attributable Disease: A Report of the Surgeon General. Atlanta, Centers for Disease Control and Prevention, 2010.

-31 Liu S, Serdula MK, Williamson DF, Mokdad AH, Byers T: A prospective study of alcohol intake and change in body weight among US adults. Am J Epidemiol 1994;140:912-920.

-32 Bagnardi V, Rota M, Botteri E, Tramacere I, Islami F, Fedirko V, Scotti L, Jenab M, Turati F, Pasquali E, Pelucchi C, Galeone C, Bellocco R, Negri E, Corrao G, Boffetta P, La Vecchia C: Alcohol consumption and site-specific cancer risk: a comprehensive dose-response meta-analysis. Br J Cancer 2015;112:580-593.

33 Nyholm M, Gullberg B, Merlo J, Lundqvist-Persson C, Rastam L, Lindblad U: The validity of obesity based on self-reported weight and height: Implications for population studies. Obesity (Silver Spring) 2007;15:197208.

-34 Skeie G, Mode N, Henningsen M, Borch KB: Validity of self-reported body mass index among middle-aged participants in the Norwegian Women and Cancer study. Clin Epidemiol 2015;7:313-323.

-35 Borch KB, Ekelund U, Brage S, Lund E: Criterion validity of a 10-category scale for ranking physical activity in Norwegian women. Int J Behav Nutr Phys Act 2012;9:2.

36 Willett WC: Nutritional Epidemiology, 2nd ed. New York, Oxford University Press, 1998. 